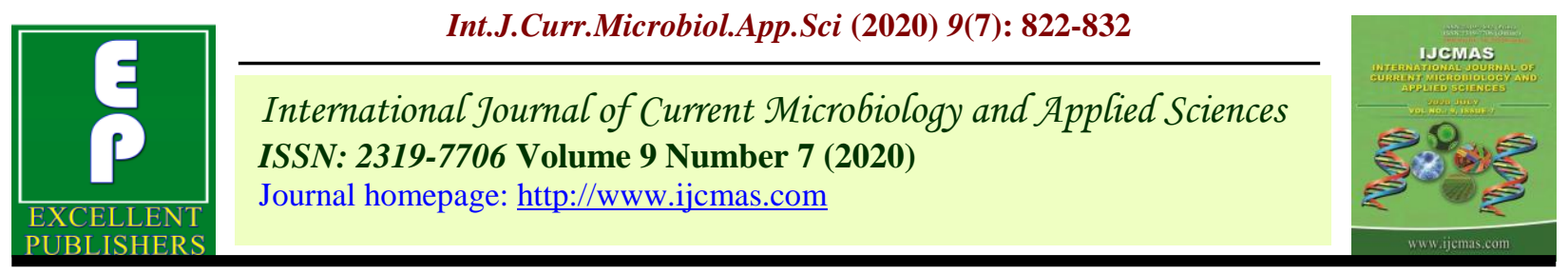

Original Research Article

https://doi.org/10.20546/ijcmas.2020.907.095

\title{
ISSR Marker Based Genetic Diversity Analysis of 35 Garcinia Accessions (Garcinia gummi-Gutta (L) Roxb)
}

\author{
Anu G. Krishnan*, Anu Cyriac, K. Bini, Sible George Varghese \\ Regional Agricultural Research Station, Kumarakom, Kottayam, Kerala, India \\ *Corresponding author
}

\section{A B S T R A C T}

\begin{tabular}{|l|}
\hline Ke y w or d s \\
Gamboge, Garcinia \\
gummi-gutta, \\
ISSR, Molecular \\
markers, Genetic \\
diversity
\end{tabular}

Genetic diversity was assessed for 35 garcinia (Garcinia gummi-gutta (L) Roxb) germplasm maintained at Regional Agricultural Research Station, Kumarakom collected from different geographical locations using ISSR markers. Out of the 34 ISSR primers screened, 18 primers were polymorphic and used for further diversity analysis. The products were detected by electrophoresis and analysed using the program NTSYS-PC. Seventy two loci were polymorphic out of the 99 loci amplified. The average Polymorphic Information Content (PIC) obtained for 18 primers was 0.33 and the average marker index was found to be 1.3. Two major clusters were identified at $64 \%$ similarity. The clustering pattern did not follow any geographic distribution pattern. The genetic similarity index ranged from 0.55 to 0.95 indicating a genetic diversity of $45 \%$ within the germplasm accessions.

\section{Introduction}

Garcinia gummi-gutta (L) Roxb., popularly known as Camboge (Gaertn.) Desr. or Malabar tamarind (Kudampuli) belongs to the family Clusiaceae. The family consists of nearly 200 species and shows a very high degree of diversity (Osman and Rahman 2006). Among the members G.gummigutta $(L$.$) is originated from Indonesia. It is an$ endemic, fruit yielding tree species widely distributed in the Western Ghats of India. Recently the plant is having very high importance in the commercial market due to the presence of hydroxy citric acid in the fruit rind and leaves of the plant, which is reported to be an anti- obesity compound (Shara et al., 2004; Saito et al., 2005).

Moreover in India, the fruit rind is used as a sour flavoring spice in preparation of many dishes. In this context strengthening of research on genetic diversity of Garcinia gummy-gutta has great scientific importance for the conservation of germplasm resources. Characterization of germplasm is important 
for the effective utilization and conservation of the genotypes. Molecular markers such as RAPD, ISSR and SSR markers are very efficient tools for genotyping. The most important character of the DNA markers is that they are not affected by environmental factors and the variation (polymorphism) can be detected at DNA level using simple technologies. The micro satellite based molecular marker system, Inter-sample sequence repeat (ISSR) developed by Zietkiewicz et al., (1994), provides ample polymorphism, high reproductibility, great stability, simple operation, low cost, low DNA usage and safety. Parthasarathy et al., (2013) reported that both ISSR markers and RAPD are very effective in Garcinia to study about the diversity. Earlier Tharachand et al., (2015) observed a high level of genetic diversity among the genotypes studied using RAPD markers. He stated that the data is supported by the large variations observed in the morphological features of the sampled accessions. Recently Ravishankar et al., (2017) developed a huge number of SSR markers for Garcinia using the next generation sequencing technologies. They have selected randomly 32 numbers of markers and tested in thirty accessions of Garcinia gummi-gutta. The markers were very effective and the number of alleles per locus ranges from 12-27. It reflects the high level of heterozygosity among the genotypes. The present study was aimed to analyze the genetic diversity among 35 accessions of Camboge using ISSR markers which may be useful for further utilization of germplasm and genetic breeding.

\section{Materials and Methods}

\section{Plant materials and DNA isolation}

Thirty five superior genotypes of ' Kudampuli' which were identified from the field germplasm maintained at RARS, Kumarakom were exploited in this study (Table 1). These were collected from different geographical locations of Kerala, in Kottayam, Alappuzha and Pathanamthitta districts. DNA was isolated in the laboratory of Regional Agricltural Research Station, Kumarakom using the modified Doyle and Doyle CTAB DNA extraction method. The quantity of the DNA isolated was checked using the Qubit fluorometer and the quality was assured based on the 260/280 ratio. It was further checked on a $0.8 \% \mathrm{w} / \mathrm{v}$ agarose gel. The working sample of each DNA was adjusted to 50 ng/microlitre

\section{Amplification of ISSR primers}

A total of 34 ISSR primers in UBC series(UBC 900) synthesised by Sigma genosys which were reported in the studies of Parthasarathy et al., 2016 were tested for their amplification in 35 accessions of garcinia. The PCR reactions were carried out in a Agilent Thermocycler with $25 \mu \mathrm{l}$ reaction volume which contains $1 \mathrm{X}$ PCR buffer, 1.5 $\mathrm{mM} \mathrm{MgCl}_{2}, 400 \mu \mathrm{MdNTPs}$, 50ng template DNA, 10 picomol primer and 1u Taq DNA polymerase (TaKaRa). The PCR program consisted of 35 cycles with an initial denaturation of $94^{\circ} \mathrm{C}$ for $2 \mathrm{~min}$. Each cycle was programmed for a denaturation at $94^{\circ} \mathrm{C}$ for 40 seconds, annealing temperature of $50^{\circ} \mathrm{C}$ for 1 minute and the primer elongation at $72^{\circ} \mathrm{C}$ for $1.5 \mathrm{~min}$. The final primer extension was set at $72^{\circ} \mathrm{C}$ for 10 minutes and hold at $4^{0} \mathrm{C}$ till the tubes are taken. Amplified DNA was resolved in a $1.5 \%$ Agarose (Sigma) gel along with a $1 \mathrm{~kb}$ DNA ladder (Thermo Scientific).

\section{Data analysis and scoring}

Only clear and reproducible bands were considered for scoring. The data were scored from the gel images using ' 1 ' for presence and ' 0 ' for absence of band. 
The polymorphic information content for each marker was calculated using the formula: PIC $=1-($ Weir 1990), where Pi is the frequency of the $i^{\text {th }}$ allele in the genotypes studied. For dominant markers, this formula can be simplified to $\mathrm{PIC}=2 \mathrm{PiQi}$ (Roldan-Ruiz et al., 2000) where $\mathrm{Pi}$ is the frequency of presence and Qi is the frequency of absence of a particular band and the maximum value is 0.5 . To calculate PIC value for a primer, the PIC values for all the polymorphic bands produced by the primer were averaged (Rana and Bhat 2004; Tehrani et al., 2008). Marker index were calculated by multiplying PIC with number of polymorphic bands(Powell et al., 1996). For the assessment of genetic diversity, jaccards similarity coefficient was used to calculate the similarity between sample sets for generating similarity matrix. The cluster analysis was performed using the unweighted pair group method for arithmetic mean (UPGMA) and the dendrogram was generated using the program NTSYS-PC.

\section{Results and Discussion}

\section{ISSR analysis}

Out of random 34 ISSR primers tested, 18 primers produced clear and consistent polymorphism among the 35 garcinia accessions. All these ISSR primers were composed of di nucliotide repeat sequences (Table 1). Fig.1 shows the banding pattern of 35 garcinia accessions using ISSR primers UBC 848a and TC10G. A total of 99 DNA loci were generated, out of which 72 bands were polymorphic and 27 were monomorphic. The polymorphic loci percentage obtained in this study was $72.7 \%$. The number of loci amplified ranged from two to thirteen with an average of 5.5 loci per marker (Table 2). The number of polymorphic loci ranged between 1 to 12 and the ratio of polymorphic loci was between $50 \%$ to $87.5 \%$ with an average of $72.7 \%$ which indicates the effectiveness of
ISSR markers. Average number of DNA fragments amplified by ISSR primers in this study was slightly lower when compared to the ISSR amplication reported in G. mangostana and garcinia spp by Sobir et al., 2011, which was 6.1. The marker 840a amplified highest number of loci (13) of them $12(92.3 \%)$ were polymorphic. Out of the 99 bands scored four were genotype specific. The polymorphic information content (PIC) value ranged from 0.08 to 0.46 . The highest and lowest PIC values were observed for the markers TC10G and 841a respectively. The marker index (MI) for the primers were ranged from $0.24(841 \mathrm{a})$ to $3.06(843 \mathrm{a})$ with an average of 1.3. ISSR markers have also been successfully used for genetic variability studies in garcinia spp (Sobir et al., 2011, Parthasarathy et al., 2013). In a study on mangosteen diversity using ISSR markers Mansyah et al., (2010) obtained 58\% polymorphic bands out of 72 bands generated by 11 random ISSR primers in 23 accessions. In the present study 18 ISSR markers generated 72 polymorphic bands with an average of 4 bands per primer. Which is comparable with 3.82 bands on study of Garcinia mangostana by Mansyah et al., (2010).

The amplification patterns of different ISSR primers were different in this study and these ISSR primers effectively revealed the genetic diversity among the studied garcinia accessions.

\section{Genetic relationship analysis}

Genetic diversity analysis was carried out based on the dominant scoring of the data. The similarity index values were ranged from 0.55 to 0.95 (Table 3 ) indicating significant level of diversity among the accessions studied. Maximum similarity was observed for acc. no. 13/90 (181) and 15/90 (194 Amrutham). 
Table.1 Garcinia gummi-gutta genotypes used for the study

\begin{tabular}{|c|c|c|c|}
\hline Si.No. & Accession no. & Tree no. & Place of collection \\
\hline 1 & $3 / 88$ & 252 & Kavumbhagam, Mannankarachira \\
\hline 2 & 3/91 & 250 & Kavumbhagam, Mannankarachira \\
\hline 3 & $3 / 93$ & 247 & Manippuzha \\
\hline 4 & $3 / 108$ & 254 & Alumthuruthy \\
\hline 5 & $3 / 116$ & 248 & Podiyadi \\
\hline 6 & $3 / 137$ & 144 & Melukara, Kozhencherry \\
\hline 7 & $3 / 130$ & 65 & Varayanoor, Pullad \\
\hline 8 & $3 / 132$ & 24 & Chenganoor \\
\hline 9 & $4 / 151$ & 234 & Perublem \\
\hline 10 & $4 / 154$ & 139 & Perublem \\
\hline 11 & $4 / 156$ & 209 & Perublem \\
\hline 12 & $4 / 172$ & 170 & Perlassery \\
\hline 13 & $4 / 177$ & 233 & Mahadevikkad \\
\hline 14 & $4 / 160$ & 10 & Thalavadi \\
\hline 15 & $4 / 187$ & 141 & Kelamangalam, Thakazhi \\
\hline 16 & $5 / 15$ & 114 & Kummanam \\
\hline 17 & $5 / 19$ & 116 & Kummanam \\
\hline 18 & $5 / 31$ & 47 & Olassa \\
\hline 19 & $5 / 36$ & 9 & Udayanapuram \\
\hline 20 & $5 / 66$ & 164 & Nadakkal \\
\hline 21 & $64 / 90$ & 173(Haritham) & Madapally, Vaikom \\
\hline 22 & $4-\mathrm{A} / 90$ & 174 & Attipeedika,Kumarakom \\
\hline 23 & $2 / 90$ & 176 & Attipeedika, Kumarakom \\
\hline 24 & $4 / 90$ & 178 & Attipeedika, Kumarakom \\
\hline 25 & $6 / 90$ & 180 & Alumchottil \\
\hline 26 & $13 / 90$ & 181 & Olassa \\
\hline 27 & $16 / 90$ & 183 & Olassa \\
\hline 28 & $10 / 90$ & 185 & Olassa \\
\hline 29 & 9-D/90 & 187 & Olassa \\
\hline 30 & $4 / 1 / 90$ & 189 & Edathua \\
\hline 31 & $45 / 90$ & 191(Nithya) & Edathua \\
\hline 32 & $33 / 90$ & 193 & Valanjavattom, Pathanamthitta \\
\hline 33 & $15 / 90$ & 194(Amrutham) & Olassa \\
\hline 34 & $16 \mathrm{~A} / 90$ & 196 & Olassa \\
\hline 35 & $22 / 90$ & 197 & Thiruvarppu \\
\hline
\end{tabular}


Table.2 Characteristics of polymorphic ISSR markers amplified in Garcinia

\begin{tabular}{|c|c|c|c|c|c|c|}
\hline S.No. & Primer & $\begin{array}{l}\text { Total no. of } \\
\text { bands amplified }\end{array}$ & $\begin{array}{l}\text { Number of } \\
\text { polymorphic bands }\end{array}$ & $\begin{array}{l}\text { Percentage of } \\
\text { polymorphism }\end{array}$ & PIC & MI \\
\hline 1 & 807 & 4 & 2 & 50 & 0.36 & 0.72 \\
\hline 2 & 809 & 6 & 3 & 50 & 0.19 & 0.57 \\
\hline 3 & 810 & 4 & 2 & 50 & 0.42 & 0.84 \\
\hline 4 & 812 & 3 & 3 & 100 & 0.38 & 1.14 \\
\hline 5 & 835 & 4 & 2 & 50 & 0.28 & 0.56 \\
\hline 6 & $836 a$ & 3 & 2 & 66.6 & 0.29 & 0.58 \\
\hline 7 & $840 \mathrm{a}$ & 13 & 12 & 92.3 & 0.25 & 3.0 \\
\hline 8 & $840 b$ & 6 & 4 & 66.6 & 0.30 & 1.2 \\
\hline 9 & $841 \mathrm{a}$ & 3 & 2 & 66.6 & 0.08 & 0.24 \\
\hline 10 & $841 \mathrm{~b}$ & 5 & 3 & 60 & 0.35 & 1.75 \\
\hline 11 & $842 b$ & 7 & 6 & 85.7 & 0.36 & 2.52 \\
\hline 12 & $843 a$ & 9 & 7 & 77.7 & 0.34 & 3.06 \\
\hline 13 & $844 a$ & 7 & 4 & 57.1 & 0.38 & 1.52 \\
\hline 14 & $848 \mathrm{a}$ & 8 & 7 & 87.5 & 0.35 & 2.45 \\
\hline 15 & $850 \mathrm{a}$ & 8 & 7 & 87.5 & 0.28 & 1.96 \\
\hline 16 & $857 \mathrm{~b}$ & 2 & 1 & 50 & 0.45 & 0.45 \\
\hline 17 & 860 & 2 & 1 & 50 & 0.45 & 0.45 \\
\hline \multirow[t]{2}{*}{18} & $\mathrm{TC} 10 \mathrm{G}$ & 5 & 4 & 80 & 0.46 & 1.84 \\
\hline & Total/Average & $99(5.5)$ & $72(4)$ & 72.7 & 0.33 & 1.3 \\
\hline
\end{tabular}


Table.3 Genetic similarity coefficient between Garcinia accessions

\begin{tabular}{|c|c|c|c|c|c|c|c|c|c|c|c|c|c|c|c|c|c|}
\hline 1 & 2 & 3 & 4 & 6 & 7 & 8 & 9 & 10 & 11 & 13 & 14 & 15 & 16 & 17 & & & \\
\hline 1 & 1.000 & & & & & & & & & & & & & & & & \\
\hline 2 & 0.671 & 1.000 & & & & & & & & & & & & & & & \\
\hline 3 & 0.681 & 0.679 & 1.000 & & & & & & & & & & & & & & \\
\hline 4 & 0.766 & 0.737 & 0.688 & 1.000 & & & & & & & & & & & & & \\
\hline 5 & 0.656 & 0.662 & 0.644 & 0.714 & 1.000 & & & & & & & & & & & & \\
\hline 6 & 0.650 & 0.580 & 0.617 & 0.650 & 0.648 & 1.000 & & & & & & & & & & & \\
\hline 7 & 0.763 & 0.757 & 0.671 & 0.847 & 0.686 & 0.654 & 1.000 & & & & & & & & & & \\
\hline 8 & 0.691 & 0.671 & 0.700 & 0.722 & 0.681 & 0.663 & 0.696 & 1.000 & & & & & & & & & \\
\hline 9 & 0.740 & 0.806 & 0.642 & 0.696 & 0.690 & 0.566 & 0.671 & 0.718 & 1.000 & & & & & & & & \\
\hline 10 & 0.701 & 0.726 & 0.688 & 0.711 & 0.667 & 0.628 & 0.662 & 0.718 & 0.707 & 71.000 & & & & & & & \\
\hline 11 & 0.679 & 0.692 & 0.686 & 0.659 & 0.649 & 0.553 & 0.622 & 0.722 & 0.718 & 0.757 & 1.000 & & & & & & \\
\hline 12 & 0.650 & 0.707 & 0.676 & 0.750 & 0.671 & 0.609 & 0.711 & 0.688 & 0.747 & 70.726 & 0.724 & 1.000 & & & & & \\
\hline 13 & 0.722 & 0.737 & 0.753 & 0.744 & 0.690 & 0.610 & 0.705 & 0.639 & 0.675 & 50.711 & 0.744 & 0.770 & 1.000 & & & & \\
\hline 14 & 0.720 & 0.736 & 0.707 & 0.720 & 0.701 & 0.689 & 0.693 & 0.675 & 0.693 & 0.732 & 0.675 & 0.689 & 0.743 & 1.000 & & & \\
\hline 15 & 0.788 & 0.738 & 0.711 & 0.702 & 0.641 & 0.616 & 0.688 & 0.702 & 0.741 & 0.803 & 0.702 & 0.725 & 0.744 & 0.700 & 1.000 & & \\
\hline 16 & 0.707 & 0.700 & 0.635 & 0.687 & 0.680 & 0.619 & 0.691 & 0.785 & 0.704 & 0.709 & 0.728 & 0.731 & 0.707 & 0.705 & 0.750 & 1.000 & \\
\hline 17 & 0.731 & 0.770 & 0.654 & 0.731 & 0.681 & 0.638 & 0.705 & 0.722 & 0.773 & 0.711 & 0.709 & 0.701 & 0.709 & 0.753 & 0.732 & 0.829 & 1.000 \\
\hline 18 & 0.725 & 0.740 & 0.734 & 0.663 & 0.632 & 0.654 & 0.688 & 0.738 & 0.659 & 0.684 & 0.704 & 0.692 & 0.725 & 0.701 & 0.706 & 0.821 & 0.756 \\
\hline 19 & 0.684 & 0.767 & 0.760 & 0.705 & 0.725 & 0.675 & 0.711 & 0.727 & 0.701 & 0.764 & 0.684 & 0.716 & 0.750 & 0.800 & 0.750 & 0.756 & 0.760 \\
\hline 20 & 0.692 & 0.684 & 0.724 & 0.737 & 0.686 & 0.662 & 0.697 & 0.692 & 0.646 & 0.775 & 0.692 & 0.649 & 0.714 & 0.736 & 0.695 & 0.659 & 0.658 \\
\hline 21 & 0.642 & 0.633 & 0.737 & 0.684 & 0.710 & 0.654 & 0.688 & 0.763 & 0.658 & 0.684 & 0.727 & 0.697 & 0.663 & 0.703 & 0.628 & 0.734 & 0.671 \\
\hline 22 & 0.741 & 0.734 & 0.750 & 0.785 & 0.761 & 0.612 & 0.747 & 0.808 & 0.738 & 0.753 & 0.741 & 0.753 & 0.785 & 0.740 & 0.783 & 0.790 & 0.728 \\
\hline 23 & 0.691 & 0.622 & 0.700 & 0.713 & 0.635 & 0.666 & 0.676 & 0.757 & 0.688 & 0.747 & 0.734 & 0.760 & 0.734 & 0.688 & 0.714 & 0.748 & 0.659 \\
\hline 24 & 0.750 & 0.718 & 0.658 & 0.689 & 0.727 & 0.584 & 0.699 & 0.712 & 0.708 & 0.776 & 0.703 & 0.671 & 0.680 & 0.779 & 0.737 & 0.757 & 0.736 \\
\hline 25 & 0.692 & 0.707 & 0.658 & 0.737 & 0.671 & 0.662 & 0.720 & 0.705 & 0.646 & 0.716 & 0.714 & 0.693 & 0.737 & 0.667 & 0.655 & 0.722 & 0.724 \\
\hline 26 & 0.716 & 0.667 & 0.704 & 0.759 & 0.653 & 0.709 & 0.722 & 0.667 & 0.651 & 0.718 & 0.655 & 0.722 & 0.738 & 0.737 & 0.678 & 0.682 & 0.704 \\
\hline 27 & 0.747 & 0.720 & 0.714 & 0.747 & 0.634 & 0.600 & 0.730 & 0.650 & 0.675 & 0.764 & 0.737 & 0.740 & 0.773 & 0.703 & 0.738 & 0.700 & 0.724 \\
\hline 28 & 0.718 & 0.757 & 0.705 & 0.787 & 0.686 & 0.605 & 0.795 & 0.667 & 0.692 & 0.720 & 0.614 & 0.781 & 0.740 & 0.740 & 0.741 & 0.683 & 0.750 \\
\hline 29 & 0.605 & 0.616 & 0.667 & 0.658 & 0.636 & 0.595 & 0.630 & 0.704 & 0.600 & 0.671 & 0.704 & 0.639 & 0.649 & 0.611 & 0.600 & 0.689 & 0.694 \\
\hline 30 & 0.778 & 0.689 & 0.658 & 0.730 & 0.677 & 0.722 & 0.693 & 0.662 & 0.703 & 0.662 & 0.649 & 0.736 & 0.730 & 0.718 & 0.718 & 0.737 & 0.778 \\
\hline
\end{tabular}




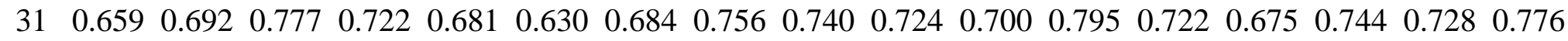
$\begin{array}{llllllllllllllllll}32 & 0.803 & 0.708 & 0.703 & 0.703 & 0.647 & 0.653 & 0.703 & 0.720 & 0.736 & 0.712 & 0.726 & 0.676 & 0.740 & 0.729 & 0.727 & 0.770 & 0.740\end{array}$

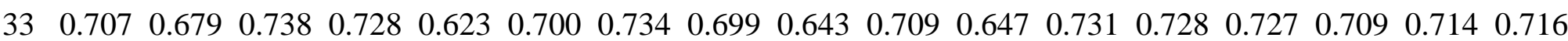

$\begin{array}{lllllllllllllllllll}34 & 0.627 & 0.591 & 0.627 & 0.646 & 0.574 & 0.641 & 0.631 & 0.612 & 0.597 & 0.625 & 0.703 & 0.694 & 0.652 & 0.641 & 0.562 & 0.647 & 0.636\end{array}$

$\begin{array}{lllllllllllllllllll}35 & 0.773 & 0.654 & 0.714 & 0.705 & 0.639 & 0.633 & 0.720 & 0.654 & 0.638 & 0.684 & 0.642 & 0.707 & 0.750 & 0.726 & 0.728 & 0.691 & 0.692\end{array}$

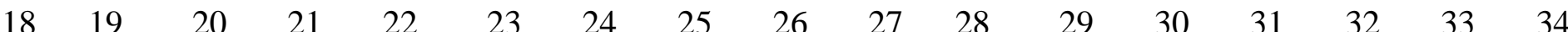$$
1
$$$$
2
$$$$
3
$$$$
4
$$$$
5
$$$$
6
$$$$
7
$$$$
8
$$$$
9
$$

10

11

12

13

14

15

16

17

181.000

$\begin{array}{lll}19 & 0.753 & 1.000\end{array}$

$\begin{array}{llll}20 & 0.675 & 0.817 & 1.000\end{array}$

$\begin{array}{lllll}21 & 0.709 & 0.646 & 0.654 & 1.000\end{array}$

$\begin{array}{llllll}22 & 0.744 & 0.769 & 0.713 & 0.792 & 1.000\end{array}$

$\begin{array}{lllllll}23 & 0.716 & 0.718 & 0.727 & 0.787 & 0.753 & 1.000\end{array}$

$\begin{array}{llllllll}24 & 0.707 & 0.809 & 0.743 & 0.645 & 0.724 & 0.680 & 1.000\end{array}$

$\begin{array}{llllllllll}25 & 0.718 & 0.768 & 0.753 & 0.675 & 0.713 & 0.705 & 0.686 & 1.000\end{array}$

$\begin{array}{lllllllllll}26 & 0.720 & 0.700 & 0.688 & 0.679 & 0.674 & 0.750 & 0.707 & 0.667 & 1.000\end{array}$

$\begin{array}{lllllllllll}27 & 0.753 & 0.733 & 0.697 & 0.684 & 0.744 & 0.727 & 0.732 & 0.684 & 0.731 & 1.000\end{array}$ 


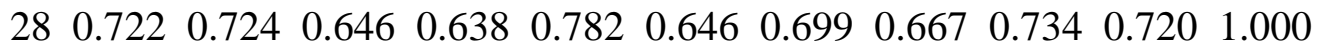

$\begin{array}{llllllllllllll}29 & 0.708 & 0.700 & 0.671 & 0.671 & 0.712 & 0.694 & 0.662 & 0.639 & 0.653 & 0.686 & 0.644 & 1.000\end{array}$

$\begin{array}{llllllllllllll}30 & 0.781 & 0.690 & 0.653 & 0.676 & 0.714 & 0.707 & 0.676 & 0.746 & 0.747 & 0.699 & 0.750 & 0.639 & 1.000\end{array}$

$\begin{array}{lllllllllllllllll}31 & 0.725 & 0.750 & 0.650 & 0.750 & 0.808 & 0.713 & 0.667 & 0.671 & 0.716 & 0.760 & 0.740 & 0.704 & 0.740 & 1.000\end{array}$

$\begin{array}{llllllllllllllllll}32 & 0.870 & 0.746 & 0.718 & 0.685 & 0.757 & 0.764 & 0.761 & 0.676 & 0.831 & 0.797 & 0.736 & 0.676 & 0.746 & 0.716 & 1.000\end{array}$

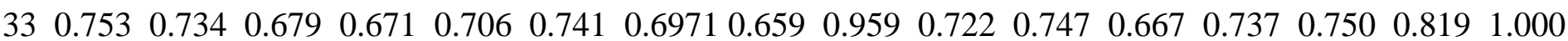

$\begin{array}{llllllllllllllllll}34 & 0.667 & 0.631 & 0.694 & 0.672 & 0.672 & 0.677 & 0.597 & 0.651 & 0.677 & 0.683 & 0.656 & 0.672 & 0.694 & 0.636 & 0.688 & 0.692 & 1.000\end{array}$

$\begin{array}{lllllllllllllllllll}35 & 0.731 & 0.757 & 0.720 & 0.625 & 0.725 & 0.718 & 0.708 & 0.675 & 0.753 & 0.675 & 0.819 & 0.686 & 0.746 & 0.705 & 0.809 & 0.766 & 0.667 & 1.000\end{array}$

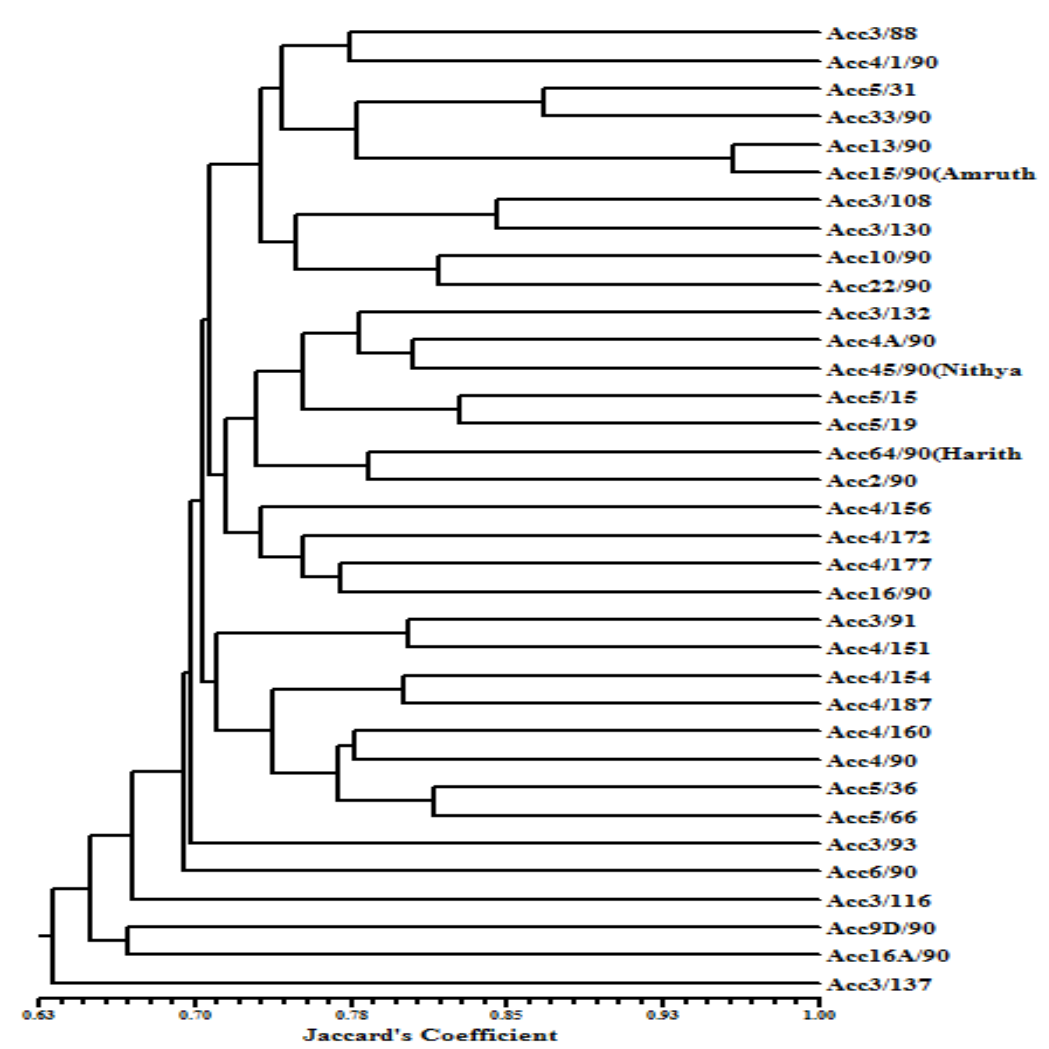

Fig.1 Dendrogram showing genetic relationship between 35 garcinia accessions 


\section{1234556789101112131415161718 M 1920212223242526272829303132333435}

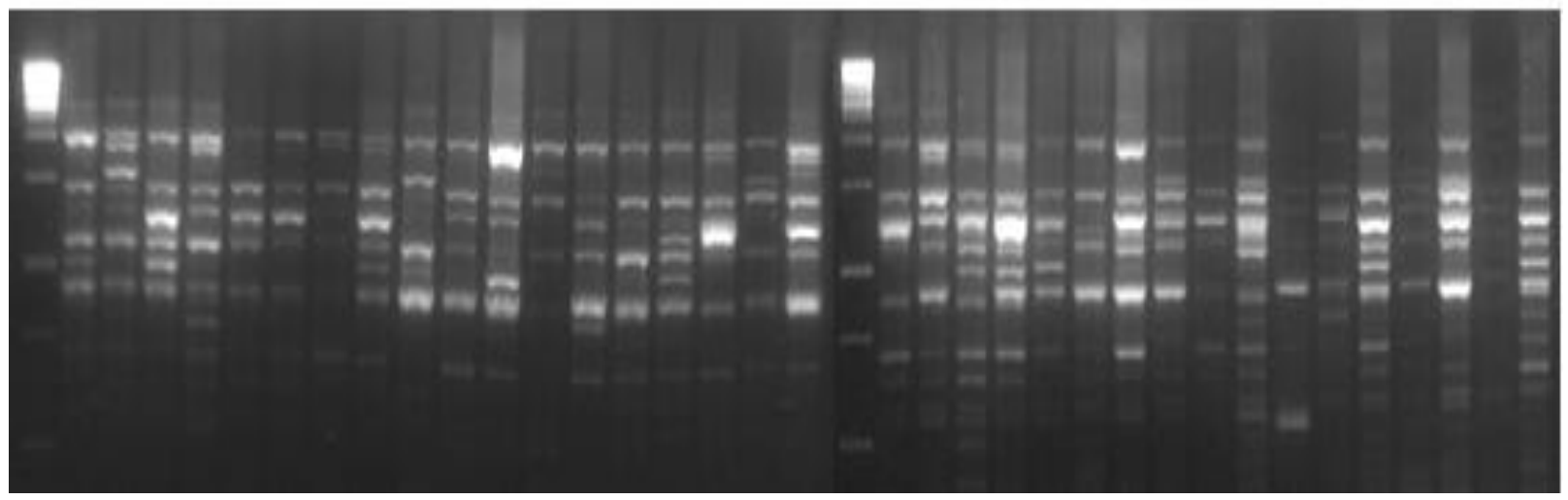

Fig.2 ISSR profiling of selected Garcinia genotypes using UBC848a

UBC848a

Lane M: 1 Kb DNA Ladder; Lane 1: Acc. 252; Lane2: Acc. 250; Lane 3:Acc.247; Lane 4 Acc. 254; Lane 5 : Acc.248; Lane 6 : Acc.144; Lane 7 : Acc.65; Lane 8: Acc.24; Lane 9: Acc.234; Lane 10: Acc.139; Lane 11: Acc.209; Lane 12: Acc.170; Lane 13: Acc.233; Lane 14: Acc.10; Lane 15: Acc.141; Lane 16: Acc.114; Lane 17: Acc.116; Lane 18: Acc.47; Lane 19: Acc.9; Lane 20: Acc.164; Lane 21: Acc.173 (Haritham); Lane 22: Acc.174; Lane 23: Acc.176; Lane 24: Acc.178; Lane 25: Acc.180; Lane 26: Acc.181; Lane 27: Acc.183; Lane 28: Acc.185; Lane 29: Acc.187; Lane 30: Acc.189; Lane 31: Acc.191; Lane 32: Acc.193; Lane 33: Acc.194; Lane 34: Acc.196; Lane 35: Acc.197

UPGMA cluster analysis separated the 35 accessions mainly into two major clusters at $64 \%$ similarity. Cluster II contained only one genotype, Acc.no. 3/137 and all other genotypes were grouped into cluster I. Cluster I was further divided into six sub clusters at $70 \%$ similarity.

The sixth subcluster was again divided to 5 major clusters at $0.72 \%$ similarity. Among that acc.no.3/93 (248), 6/90 (180) and 3/ 116 (247) were formed separate individual clads. The released varieties Haritham and Nithya were grouped in to same subcluster whereas the variety Amritam was placed in a different subcluster.

The Haritham and Nithya are grouped as varieties suitable for homestead cultivation while Amrutham is considered as suitable for commercial cultivation. These were the high yielding varieties released from Kerala Agricultural University and they are grouped under one major cluster. The maximum similarity for Haritham was observed with the acc.no. 2/90 (79\%). The variety Nithya has maximum similarity with the acc.4A/90 (80\%) and Amritam showed 95\% similarity with the Acc 13/90.

The genotype 3/137 which was separated alone forming the cluster II was collected from a different geographical location viz. Kozhencherry. The clustering pattern did not follow any geographic distribution pattern. Similar results were obtained for mangosteen accessions where clustering pattern did not represent their origin (Sinaga, 2008; Sobir et al., 2011). According to Murthy and Arunachalam (1966) genetic drift and selection in different environments can cause greater diversity among genotypes than geographic distance. Hence selection of parental material based in geographic diversity in breeding programmes may not be effective.

The result of this study indicates that 18 ISSR 
primers used were suitable for the genetic diversity analysis of garcinia as they were highly polymorphic. The findings also showed that there exists a great amount of diversity among the 35 garcinia accessions.

\section{References}

Mansyah, E., Sobir, S., Santosa, E., and Roedhy, P. 2010. Assessment of inter simple sequence repeat (ISSR) techniques in mangosteen (Garcinia mangostana L) grown in different Sumatra region. J. Hortic For. 2(6): 127-134.

Murty, B. R., and Arunachalam, V. 1966. The nature of divergence in relation to breeding systems in some crop plants. Indian J. Gent. Pl.Breed. 26 : 188-198.

Osman, M. B., and Milan, A. R. 2006. Mangosteen: Garcinia mangostana L. Williams, J.T., Smith, R.W., Haq, N. and Dunsiger, Z. (eds.). In: Fruits for the future. Southampton, UK. 170pp.

Parthasarathy, U., Nandakishore, O.P., Babu, N.K., Kumar, S., and Parthasarathy, V.A. (2013). Comparative effectiveness of inter-simple sequence repeat and randomly amplified polymorphic DNA markers to study genetic diversity of Indian Garcinia. Afr. J. Biotechnol., 12(46):6443-6451.

Parthasarathy, U., Nandakishore, O.P., Rosana, O.B., Babu, K.N., Kumar, R.S., Parthasarathy, V.A. 2016. Indian J Exp Biol. 54(6):400-405.

Powell, W., Morgante, M., Andre, C. 1996. The comparison of RFLP, RAPD, AFLP and SSR (microsatellite) markers for germplasm analysis. Mol Breeding 2: 225-238.

Rana, M. K., and Bhat, K. V. 2004. A Comparison of AFLP and RAPD Markers for Genetic Diversity and Cultivar Identification in Cotton. $J . P l$.
Biochem and Biotechnol. 13(1), 19-24.

Ravishankar, K. V., Vasudeva, R., hemanth, B., sandya, B. S., Sthapit, B. R. Parthasarathy, V. A., and Rao, V. R. 2017. Isolation and characterization of microsatellite markers in Garcinia gummi-gutta by next-generation sequencing and cross-species amplification. J. Genet. 96(2): 213218.

Roldán-Ruiz, I., Dendauw, J., and Van Bockstaele, E. (2000).AFLP markers reveal high polymorphic rates in ryegrasses (Lolium spp.). $\mathrm{Mol}$ Breed. 6:125-134.

Saito, M., Ueno, M., Ogino, S., Kubo, K., Nagata, J. and Takeuchi, M. 2005. High dose of cambogiais effective in suppressing fat accumulation in developing male Zucker obese rats, but highly toxic to the testis. Food Chemi. Toxic. 43: 411-419.

Shara, M., Ohia, S. E., Schmidt, R. E., Yasmin, T., Zardetto-Smith A., and Kincaid A. 2004. Physico-chemical properties of a novel (-)-hydroxycitric acid extract and its effect on body weight, selected organ weights, hepatic lipid peroxidation and DNA fragmentation, hematology and clinical chemistry, and histopathological changes over a period of 90 days. Mol. Cell. Biochem. 260: 171-186.

Sinaga, S. 2008. Morphological and genetic variability analysis of mangosteen (Garcinia mangostana L.) and its close related species. Dissertation. Bogor Agricultural University. Bogor.

Sobir,S., Poerwanto,R., Santosa, E., Sinaga,S., and Mansyah, E. 2011. Genetic variability in apomictic. Mangosteen (Garcinia mangostana) and its close relatives Garcinia SPP based on ISSR markers Biodiversitas. 12(2): 59-63.

Tehrani, M.S., Mardi, M., Saeidi, H., 
Gharehyazi, B., and Assadi, M. 2008. Transferability of genomic and ESTmicrosatellites from Festuca arundinacea Schreb. to Lolium persicum Boiss. and Hohen. ex Boiss. Int J of Bot. 4:476-480.

Tharachand, C., Immanuel. S.C. and Abraham, Z. (2015). Molecular insights into the genetic diversity of Garcinia cambogia germplasm accessions. Braz. Arch. Biol.Technol., 58(5): 765-772.
Weir, B.S .1990. Genetics data Analysis, Methods for discrete population genetic data. Sunderland, USA, Sinaur Associates.

Zietkiewicz, E., Rafalski, A., and Labuda, D. 1994. Genome Fingerprinting by Simple Sequence Repeat (SSR)Anchored Polymerase Chain Reaction Amplification. Genomics. 20(2), 176183.

\section{How to cite this article:}

Anu G. Krishnan, Anu Cyriac, K. Bini, Sible George Varghese. 2020. ISSR Marker Based Genetic Diversity Analysis of 35 Garcinia Accessions (Garcinia gummi-Gutta (L) Roxb). Int.J.Curr.Microbiol.App.Sci. 9(07): 822-832. doi: https://doi.org/10.20546/ijcmas.2020.907.095 